3.6 For successful completion of the summative DOPS assessment, the trainee should be rated as 'ready for independent practice' in all items within 2 DOPS, by 2 different assessors, one of whom is not based at their current endoscopy unit

4.1 Newly certified ERCP practitioners should have a defined period of mentorship lasting a minimum period of 2 years, with provisions available for regular progress reviews, e.g. at 3-month intervals

4.2 The ongoing training requirements of newly accredited ERCP practitioners should be identified and should be encouraged to attend further training opportunities, e.g. upskilling courses

4.3 Clinicians who have recently certified in ERCP should have systems in place to ensure appropriate case load selection: regular vetting of cases or through weekly HPB MDT/triage meetings

4.4 There should be appropriate mechanisms in place for performance monitoring and review during the agreed transition period, e.g. at 3-monthly intervals

for ERCP certification: 1) performing $\geq 300$ hands-on procedures; 2) attending a JAG-accredited ERCP skills course; 3) in modified Schutz 1-2 procedures: achieving native papilla cannulation rate $\geq 80 \%$, complete bile duct clearance $\geq 70 \%$, successful stenting of distal biliary strictures $\geq 75 \%$, physically unassisted in $\geq 80 \%$ of cases; 4) 30 -day post-ERCP pancreatitis rates $\leq 5 \%$; 5) satisfactory performance in formative and summative direct observation of procedural skills (DOPS) assessments.

Conclusion JAG certification in ERCP has been developed following evidence-based consensus to quality assure training and to ultimately improve future standards of ERCP practice.

\section{PTU-82 TIME TO TACKLE TRANSITION TRAINING IN GASTROENTEROLOGY: RESULTS OF A UK SURVEY}

${ }^{1}$ Ella Mozdiak*, ${ }^{2}$ AJ Brooks, ${ }^{3} \mathrm{P}$ Narula, ${ }^{1} \mathrm{DE}$ Scarlett, ${ }^{1} \mathrm{~B}$ Kulendrarajah, ${ }^{4} \mathrm{PJ}$ Smith. ${ }^{1}$ University Hospitals North Midlands NHS Trust, Stoke On Trent, UK; ${ }^{2}$ Sheffield Teaching Hospitals NHS Foundation Trust, Sheffield, UK; ${ }^{3}$ Sheffield Childrens NHS Foundation Trust, Sheffield, UK; ${ }^{4}$ Liverpool University Hospitals NHS Foundation Trust, Liverpool, UK

\subsection{6/gutjpl-2021-BSG.382}

Introduction Inadequate transition for chronic health conditions is associated with poor disease outcomes. The training of health care professionals in developmentally appropriate healthcare in the United Kingdom (UK) has not been measured. This national survey explores experience of Adolescent and Young Person (AYP) dedicated training and service delivery, from both adult and paediatric perspective.

Methods A 30-item 'SurveyMonkey ${ }^{\circledR}$ ' questionnaire was developed by the British Society of Gastroenterology (BSG) AYP committee. This was emailed by the BSG and British Society of Paediatric Gastroenterology, Hepatology and Nutrition (BSPGHAN) to its member trainees and those within five years of Consultant appointment, between June-September 2020.

Results Of 70 respondents, $71.4 \%$ were senior trainees or $\leq 5$ years post CCT, with $54 \%$ being female. AYP training was not accessible to $58 \%$ in their current or most recent post. Of those that had prior AYP training, the majority (39\%) had been to transition clinics. A fifth felt 'at least confident' that training offered adequate experience of AYP training. Nearly half of respondents wanted AYP specific training.

Significant barriers to AYP training (figure 1) and delivery were reported including lack of resources (60\%), issues with funding e.g. who pays for the service (52\%) and a lack of local interest in service development (46\%).

Important patient related factors for successful transition were reported as ability to take medications unsupervised, patient understanding of disease and treatment.

\section{Do any of the following barriers to AYP training} apply to you?

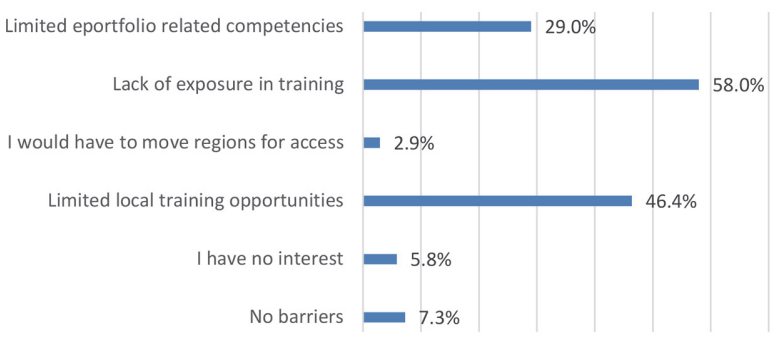

$0.0 \% 10.0 \% 20.0 \% 30.0 \% 40.0 \% 50.0 \% 60.0 \% 70.0 \%$

\section{Abstract PTU-82 Figure 1 Barriers to AYP training: responses}

Health care related barriers to successful transition $(1=$ lowest importance, $4=$ highest importance), were lack of time/ space to set up joint clinic (3.12), lack of support services (3.04) and lack of funding (3.01).

Conclusions This cross-society national survey has revealed AYP training is extremely limited and the majority of respondents perceive inadequate experience and confidence in this area, despite there being a demand for change. AYP service delivery is widely varied with lack of resources, funding and interest as key healthcare related barriers. A concerted expansion both at national curricula development level and in individual departments is urgently needed, to advance exposure and training in AYP Gastroenterology.

\section{PTU-83 THE GASTROENTEROLOGY AND HEPATOLOGY HIGHER SPECIALIST TRAINEE WORKFORCE 2020 - THE ISSUES OUR TRAINEES FACE}

${ }^{1}$ Elizabeth Ratcliffe*, ${ }^{2}$ Christopher Phillips, ${ }^{2}$ Darin Nagamootoo, ${ }^{2}$ Nina Newbery, ${ }^{3}$ Simon O'Hare, ${ }^{2}$ Nigel Trudgill, ${ }^{4}$ Charlotte Rutter. 'Wrightington Wigan And Leigh NHS Trust, Wigan, UK; ${ }^{2}$ Medical Workforce Unit Royal College of Physicians, London, UK; ${ }^{3}$ General Medical Council, London, UK; ${ }^{4}$ British Society of Gastroenterology, London, UK

\subsection{6/gutjnl-2021-BSG.383}

Introduction The COVID-19 pandemic has had a significant impact on workforce, morale and training. Even prior to this, there were considerable challenges facing gastroenterology training due to the shape of training initiative.

Methods The Medical Workforce Unit of the Royal College of Physicians (RCP) undertakes an annual census of higher specialist trainee (HST) physicians each December. Key issues affecting gastroenterology and hepatology trainees combined in the census and General Medical Council (GMC) data from 2012 - 2020 were examined. 
Results Gastroenterology national training numbers have gradually increased from 673 in 2012 to 734 in 2020. Of those who began gastroenterology training in 2012, 24\% now remain in training, 65\% have completed their CCT and 5\% have left training.

$14 \%$ of gastroenterology HSTs reported being bullied or harassed at work. Gastroenterology ranked 6th among medical specialities on this issue. Although HSTs were less likely to experience bullying or harassment than consultants, there were higher rates reported by Black, Asian and minority ethnic (BAME) than white HSTs (17\% versus 11\%). BAME HSTs were also more likely to have experienced discrimination than white HSTs (9\% versus 7\%), and women HSTs were three times as likely to experience discrimination as men $(15 \%$ versus $4 \%)$.

Gastroenterology HSTs ranked 5th most at risk of burnout among medical specialities, with rates of moderate and high burnout risk of $39 \%$ and $12 \%$ respectively. Male HSTs reported slightly greater risks of burnout than female HSTs and BAME HSTs reported markedly higher rates of high burnout risk than white HSTs (15\% versus 9\%). 62\% of HSTs said that work had impacted on their relationship with their partner and $58 \%$ with their children. $33 \%$ of trainees reported a deterioration in morale since the previous year and only $20 \%$ an improvement.

$82 \%$ of HSTs said they found their specialty work satisfying always or often, but only $22 \%$ their general internal medicine work. A smaller proportion of gastroenterology HSTs work less than full time (LTFT) than in other medical specialities (11\% versus 17\%). When asked if they wanted to train LTFT if given the opportunity, a surprising $46 \%$ of gastroenterology HSTs said they would.

Conclusions There were huge challenges facing gastroenterology HSTs before COVID-19. Worrying trends in bullying, discrimination, morale and burnout need to be addressed urgently. As time for training shortens, we must improve trainee experiences and the quality of training to protect the future gastroenterology workforce.

\section{PTU-84 ACCELERATED ACCREDITATION FOR BOWEL CANCER SCREENING COLONOSCOPIST IN CHESHIRE AND MERSEYSIDE}

${ }^{1}$ Karen Llyod, ${ }^{1}$ Carys Kinsella, ${ }^{2}$ Neil Haslam, ${ }^{3}$ Asish Bassi. ${ }^{1}$ Chesire and Merseyside Cancer Alliance, Liverpool, UK; ${ }^{2}$ Liverpool University Foundation Trust, Liverpool, UK; ${ }^{3}$ St Helens And Knowsley Nhs Trust, Prescot, UK

10.1136/gutjpl-2021-BSG.384

Introduction In 2020, as the COVID pandemic spread through the UK, Bowel Cancer screening invitations were paused. In Cheshire and Merseyside (C\& $\&$ ) there were as many as 100,000 patients waiting for their invitations. In addition, there were a large number of patients who had already been tested but waiting for colonoscopy after their positive FIT test. It is also expected that there will be an age extension to include FIT test for those aged over 50 years as the bowel scope programme is ceased. A workforce analysis suggested that there was a significant gap in the number of endoscopists needed to deliver colonoscopy across the three bowel cancer screening programmes in $\mathrm{C} \& \mathrm{M}$. There was expected to be significant delays in the accreditation process for any new endoscopist due to the suspension of training courses and assessment days.
Methods The C \& M Endoscopy Network is made up of eight Trusts on 12 sites and it is led by two Clinical Leads, a Programme Manager and a Project Manager. This Team created a project proposal to deliver Accelerated Accreditation of BCSP Colonoscopists. Funding was obtained from NHSE. The local process was approved by JAG which is the accreditation authority for the bowel cancer screening programme. This accelerated accreditation process included the development and delivery of webinars by our Clinical Lead, local assessment to replace pre accreditation courses and assessors travelling around the network to support candidates. The Mersey School of Endoscopy set up four accreditation assessment days in rapid succession and a new assessment site was added due to the efforts of the local Bowel Cancer Screening Managers. External assessors travelled across the country to support this pilot and candidates were supported by their local clinicians. New assessors were also provided training.

Results 15 candidates were put through the accelerated accreditation process to fill the workforce gap in the three BCSP across Cheshire and Merseyside over a short period of four months compared to the usual prolonged process. The Bowel Cancer screening programmes combined Clinical establishment will increase from 18 to 33 - an increase of $83 \%$. Clinicians will have honorary contracts at every Trust to allow cross trust working so that they are able to maintain the minimal numbers required for accreditation. In addition, this allowed accreditation for four additional assessors and of a new assessment site - both of which will help in future sustainability.

Conclusion This accelerated accreditation can be used a model for other programmes across the country to help with the work force gap.

\section{PTU-85 PLAYING HIDE AND SEEK: QUALITATIVE RESEARCH AND GASTROENTEROLOGY}

${ }^{1,2}$ Mohammad Farhad Peerally*, ${ }^{3}$ Vikram Mohanan, ${ }^{3}$ Ajay Verma, ${ }^{2} J o h n$ DeCaestecker. 'SAPPHIRE, Department of Health Sciences, University of Leicester, Leicester, UK; ${ }^{2}$ Digestive Diseases Centre, University Hospitals of Leicester NHS Trust, Leicester, UK; ${ }^{3}$ Digestive Diseases Unit, Kettering General Hospital NHS Foundation Trust, Kettering, UK

\subsection{6/gutjnl-2021-BSG.385}

Introduction Qualitative research includes a range of methodologies seeking to capture views of patients and practitioners on clinical interventions, and their acceptability and effectiveness in the real world. The lack of qualitative research in clinical journals, such as the BMJ, is recognised as a major concern by sections of the research community. ${ }^{1}$ No data is available on the existence of a similar issue in GI research. We seek to determine the number of original qualitative studies (including mixed-methods) published in the top $10 \mathrm{GI}$ journals, ranked according to the SCImago Journal Rank, a measure of the scientific influence of journals accounting for both citations and prestige, and identify whether qualitative research in GI is conducted in the UK, based on UK Clinical Research Network (CRN) data.

Method An advanced Pubmed search was conducted in May 2021, using a validated search strategy including a combination of terms such as 'interview', 'qualitative' and 'experience', 2 to identify qualitative studies published between 2000 2021 in the top 10 GI journals. The UK CRN database was interrogated to identify actively recruiting GI studies which include qualitative methods on the NIHR portfolio. 